\title{
Combining mouse and keyboard events with higher level desktop actions to detect mild cognitive impairment
}

\author{
Ann Gledson*, Dommy Asfiandy*, Joseph Mellor*, \\ Thamer Omer Faraj Ba-Dhfari*, Gemma Stringer ${ }^{\dagger}$, \\ Samuel Couth ${ }^{\dagger}$, Alistair Burns ${ }^{\dagger}$, Iracema Leroi ${ }^{\dagger}$, \\ Xiaojun Zeng*, John Keane*,! \\ *School of Computer Science \\ !Manchester Institute of Biotechnology \\ $\dagger$ Institute of Brain, Behaviour and Mental Health \\ University of Manchester, UK
}

\author{
Christopher Bull, Paul Rayson, Alistair Sutcliffe, \\ Peter Sawyer \\ School of Computing and Communications \\ Lancaster University, UK
}

\begin{abstract}
We present a desktop monitoring application that combines keyboard, mouse, desktop and application-level activities. It has been developed to discover differences in cognitive functioning amongst older computer users indicative of mild cognitive impairment (MCI). Following requirements capture from clinical domain experts, the tool collects all Microsoft Windows events deemed potentially useful for detecting early clinical indicators of dementia, with a view to further analysis to determine the most pertinent. Further requirements capture from potential end-users has resulted in a system that has little impact on users' daily activities and ensures data security from initial recording of events through to data analysis. We describe two experiments: firstly, volunteers were asked to perform a short set of known tasks; the second (ongoing) experiment is a longitudinal study, with the software currently successfully running on participants' computers.
\end{abstract}

Keywords-dementia, mouse dynamics, keystroke dynamics, data mining, medical informatics

\section{INTRODUCTION}

It is reported that more than 800,000 people in the UK live with dementia and this number is projected to increase to over 1 million people by 2021 [1]. Adding further to the problem, currently "only $44 \%$ of people with dementia in England, Wales and Northern Ireland receive a diagnosis" [2]; further these diagnoses are most often too late to provide optimal treatment and support, thus less effective in addressing damage to cognitive functioning. To encourage patients to present themselves for health examinations at earlier stages of diseases such as dementia, in particular Alzheimer's disease (AD) and Mild Cognitive Impairment (MCI), the older population must have an awareness of and an ability to actively engage in the detection of early clinical indicators. Promoting such selfawareness of cognitive abilities is vital to encourage selfreferral for medical assessment [3].

The number of older people using the internet is growing as email and social media fulfil the desire for increased social

Funded by the Engineering and Physical Sciences Research Counci (EPSRC) in the UK. communication, for example keeping in touch with family members [4][5]. This regular use of computers can provide opportunities to monitor older users in a pervasive and unobtrusive manner. The Software Architecture for Mental health Self-Management (SAMS) project aims to achieve this by providing:

- Non-intrusive capture of computer use - Text (e.g. email), mouse movements, keyboard typing.

- Mining of the resulting data for trends and patterns and mapping these to clinical indicators e.g. working memory and motor control.

- Inferring longitudinal changes in cognitive health, giving early indication of possible onset of dementia.

To date there is limited research in the areas of sequential event data analysis for disease detection. Much work has been done to monitor only low level mouse moves, clicks and/or keyboard strokes, omitting higher level desktop and application-level events. These are used to measure usability, to detect the effects of user impairments such as fatigue, stress or emotions on use or to distinguish between authentic users and intruders. These methods are described further in Section 2. Section 3 details the requirement elicited from end users, domain experts and data analysts for SAMS. These are expressed in terms of what is needed for the two data capture experiments undertaken in the SAMS project, both of which are dependent upon the desktop monitoring tool. Section 4 describes the design and implementation of the tool, outlining the overall framework, the technologies used to fulfil the requirements and how SAMS improves further on those outlined in Section 2 by combining mouse and keyboard events with higher level operating system events, including aggregation of events into more meaningful user tasks (e.g. delete a file by dragging it into the recycle bin). Section 5 presents several evaluation methods used to validate the output of the SAMS Monitor. Conclusions and further work are presented in the final section. 


\section{RELATED WORK}

A variety of research areas exist which look at the monitoring of electronic data to detect usage patterns.

\section{A. Data Analysis for Disease Detection}

To date little work appears to have been done in the data mining community on analysing sequential patient/user activities to detect clinical indicators of disease. In [6] multiple regression and correlation are applied to mouse movement data from 42 healthy and 20 participants with MCI in order to observe that computer mouse moves are a potential indicator of MCI. They observe that MCI participants make fewer mouse moves that were 'more variable, less efficient and with longer pauses between movements'. Both [7] and [8] observe the typing speed and accuracy, as well as mouse movement efficiency metrics recorded over a period of 6 months, to predict 16 older users' degree of cognitive health. They state that further data from a larger sample is required to draw more definitive conclusions. Although closest to the work reported here, the two studies above focus only on low level (mouse/keyboard) events and make no attempt to combine with operating system and/or application data to generate a richer data set recording higher level activities (such as dragging a folder into the recycle bin or specifying the exact icon/window on which the user has clicked).

\section{B. Mouse and Keyboard events to Measure User Impairment}

Keyboard events are monitored in [9] to detect psychomotor impairments in 14 healthy participants with the overall aim of detecting early stages of "motor-compromised neurodegenerative conditions, psychological disorders or intoxication'. Correlations were found between key-hold times and levels of psychomotor impairment when testing participants after resting and after periods of sleep deprivation. The work in [10] aims to enable interactive applications to adapt to users' contexts by measuring the 'variance in keyboard typing patterns caused by emotion'. Several features of mouse use (using clicks and movement) are used in [11] 'to assess the level of stress of students during online exams'. The exams were divided into 5 (time sequential) parts and significant differences were found between distributions for the first and final parts of the exam for around half of the participants. Again, these studies centre on low level events and omit operating system and/or application data.

\section{Mouse and Keyboard Events to Measure Usability}

Sequential pattern mining can be used on sequential data representing user activities; for example [12] use this technique to analyse user clickstreams in order to analyse how software is used. In web usability evaluation [13] use mouse movement data to find common trends that can be used to improve user interface designs. In [14] mouse and keyboard events are used to measure website usability and [15] uses mouse movements to classify user/query intent as either navigational (the user clicks quickly on a specific result) or informational (the user spends more time reading the results list). The work in [16] claims to demonstrate the 'value' of monitoring mouse movement data, by testing on two applications: (i) estimating search result relevance using mouse hovers over search result lists and (ii) differentiating between good and bad abandonment of search result pages (abandonment of a search result page without clicking any links due to dissatisfaction (bad) or finding the result directly in the results list (good)).

The ability of the above usability research to predict user satisfaction when trying to achieve particular, known goals is promising and potentially applicable to our 'set tasks' experiment. On the other hand, applying these findings to the SAMS longitudinal study where user tasks and goals are unknown (perhaps even to the participant) would be considerably more difficult.

\section{Mouse and Keyboard Events to Authenticate Users}

The classification of computer activity into genuine user or intruder, using mouse movements [17] or keystrokes [18][19] indicate that behaviour patterns can be learnt for each individual user. This suggests some potential for SAMS participants to be characterized and usage patterns analysed for differences, but leaves the question of whether any pattern detection will be sensitive enough to detect gradual changes, such as those likely in a degenerative disease such as dementia.

This section has shown a number of uses for monitoring software, and we have therefore ensured that the SAMS monitoring tool can easily be adapted to be used for such purposes in the future.

\section{REQUIREMENTS CAPTURE}

During this prototype phase, there are two direct stakeholders in the SAMS software: firstly, the end users who will eventually use the software to inform them of potential indicators of MCI; secondly, the data analysts who wish to use the data recorded by the initial version of the monitoring tool to discover the most reliable features to be used.

\section{A. End Users' Requirements}

The SAMS end-user requirements are detailed in [20] and although currently only a prototype and therefore without any alerting mechanism to warn participants of possible cognitive decline, the SAMS monitoring tool fulfils a number of requirements in order to ensure participation in our studies and to fulfil our ethical obligations to the users. The following requirements were elicited and agreed for the prototype SAMS implementation [20]):

- The ability of the user to disable the monitoring at any point, with this facility being easily accessible.

- The need for all recorded data to be private and secure, using encryption and secure transmission. In addition, a method is needed to depersonalize the data when being shared during analysis and presentation of results.

- The software is easily maintained and does not disrupt normal computer use.

- The SAMS software 'should not degrade the performance of the user's machine'. 


\section{B. Data Analysts' Requirements}

\section{1) Domain Experts}

In [21], the elicitation of requirements with the help of domain experts for the SAMS project is described. Experts in dementia, cognitive psychology and clinical research were involved in the process along with the data analysts. The process included workshops followed by questionnaires and the target was to obtain a 'causal model' for early signs of dementia. The resulting requirements are as follows [21]:

- Look for changes in activity patterns over time within each individual;

- Establish a baseline of variation for each individual, then use learning algorithms to detect changes against the baseline;

- Combine many user behaviour variables to establish the baseline and detect significant changes;

- Prioritise certain user behaviours over others, such as errors and incomplete sequences of actions;

- Benchmark individual change against known statistics for age-related change, and clinical tests of activity in people with MCI;

- Create hypothesis-directed pattern recognisers to detect problems motivated by the prioritized cognitive impairments (CIs), e.g. error classifiers in behaviour sequence analysis and excessive switching between windows. Both indicate executive dysfunction. Repeated search for the same icon/user command may also indicate a recall memory problem.

This part of the requirements capture highlighted the problem of 'unknown unknowns' [22], requirements of which neither the stakeholder nor those eliciting the requirements are aware. This suggests that where possible, it would be beneficial to collect as many user activity 'features' as possible and the resulting data mined for potential knowledge discovery.

\section{2) SAMS Experiment Design}

\section{a) Set Tasks Experiment}

This controlled experiment has been designed to compare a group of individuals with MCI $(n=22)$ with a control group of healthy participants $(n=25)$, both groups were aged 65 or over and were regular Windows users (Windows 7,8 or 10). A set of 4 Windows tasks considered typical for that age group, were followed. These were:

- General desktop operations (e.g. open a document, minimize and maximize a window, scroll to the bottom of a document, locate items and drag into folders on the desktop).

- Email tasks using Microsoft Outlook (e.g. open and reply to specified emails in the inbox).

- Word processing (e.g. open a document, edit the text by typing, cutting/pasting etc.)

- Browser tasks such as searching on Google for a specified name and then navigating around the resulting web-page by clicking on specified links and using the back button.
These tasks could take place either in the user's home or on university premises (as chosen by the participant) and identical laptops were used to prevent processing speeds or faulty equipment from affecting the results. Users were able to plug in a separate keyboard if they wished and a mouse was available for use (identical for all participants). They were also able to select either Windows 7 or 8 operating systems to be tested on. Participants were given a set of dummy tasks before starting the experiment, to familiarize them with the equipment. Once completed, the desktop monitoring tool was switched on throughout the main test and it was important that during this stage the tool remained invisible to the user and had a negligible impact on the computer's performance/speed. On the same day of the tests, a standard set of paper-based cognitive tests was also performed.

\section{b) Longitudinal Experiment}

A second experiment is currently ongoing, in which the monitoring software is installed on the participants $(n=32)$ own machines in their homes. The participants are again 65 or over and are regular Windows ( 7,8 or 10$)$ users. As this experiment is designed to look at trends within each user, the sample are either already diagnosed with early stage MCI or they are worried about their memory. The set of standard paper-based cognitive tests are performed every 4 months to monitor any changes. It is again important that the monitoring tool does not affect the participant's ability to carry out their usual computer tasks. This is both in terms of the processing and memory required to run the monitor, and also the transmission of data via the internet, to the central project server. In addition, as highlighted in Section 3.A, they must be able to easily stop and restart monitoring, with regular reminders that the software is running. The output data must be encrypted at the point of creation and remain encrypted throughout transfer to the server. Software updates should be done remotely and automatically where possible, to have minimal impact on the users' normal daily lives.

\section{3) Data Analysis Methods}

As a result of the requirements collection using domain experts, the data analysis needs to include as many variables as possible that have the potential to correspond to user behaviours. A set of data analysis techniques have been selected that are both hypothesis- and data-driven. For example, some data analysis requirements were essentially hypothesis-driven based on observations made during the 'set tasks' experiments and included:

- A count of double click errors (i.e. two consecutive single clicks that were probably meant to be double clicks).

- Frequency of 'micro drags' (very short drag movements, often made by accident, particularly when failing to double click).

- Time duration and mouse movement in pixels, between clicks.

Requests were also made for general counts and timings of all recorded desktop events during the set tasks (e.g. left/right mouse click and drag delete counts/durations; counts of mouse move into/out of icons). This is to facilitate systematic 
checking of any correlations with levels of cognitive impairment shown in the standard paper tests.

More complex data analysis methods are required for the longitudinal data, where user goals are unknown. These include variation and sequence analyses that compare sets of contiguous events that have been divided into either uniform time segments (e.g. ten minute segments) or that are processed using a sliding windows approach. Events such as mouse down, changes in focus between windows, system file changed events, windows being opened, closed, minimized or maximized and menus being opened or closed are required as input. In addition, we capture mouse hover movements in and out of desktop icons/windows, mouse movement/duration figures and keystrokes metrics divided into several categories (alpha-numeric, shift/control, back/delete, punctuation, spacing keys, arrow keys and miscellaneous keys).

\section{Requirements Discussion}

Both sets of stakeholder requirements taken together suggest the need for a balance between fulfilment of the enduser goals, which are essential for user acceptance and ethical reasons, and the capturing of as much potentially relevant data as possible to cover the unknown unknowns, that might be discovered using the data analysis methods described. This balance is also reflected at the practical level in a comparison of the experiment designs with the desired data analysis methods, again setting the need for a robust and secure monitor with negligible performance impact against a rich set of data that efficiently records event data in a format useful for complex analysis.

\section{SYSTEM DESIGN AND IMPLEMENTATION}

\section{A. Architecture}

In [23] the complete SAMS system architecture is described (including a set of browser extensions designed for a textual analysis component outside the scope of this work). This is illustrated in Figure 1.

Fig. 1. Abstract architecture of SAMS framework [23]

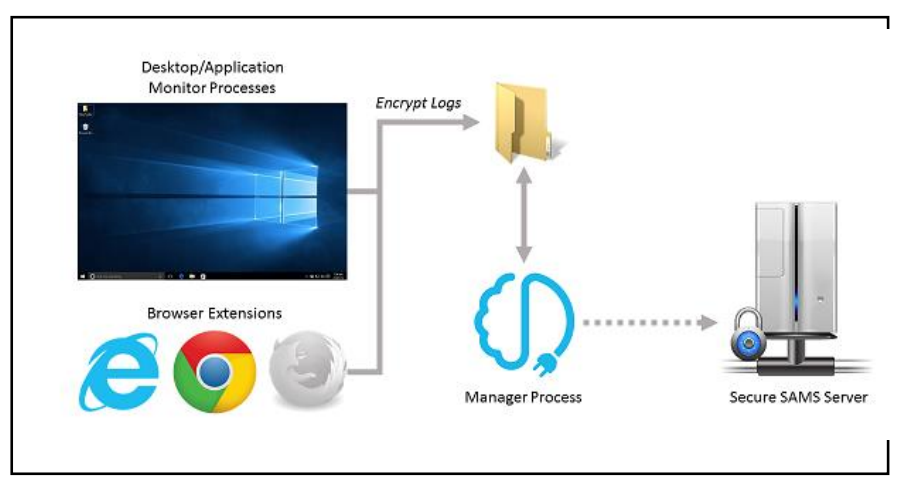

In essence, the SAMS desktop monitor converts events into text files which it encrypts before saving to a log folder. A manager component (described further in [23]) controls all user interface elements, for starting, stopping, and pausing the loggers. It is also responsible for uploading the encrypted logs to the SAMS server, and automatic update of the SAMS software.

\section{B. SAMS Monitoring component}

The desktop logger records user activities at three levels: keyboard and mouse, operating system (e.g. desktop activities) and application level. All windows events deemed potentially useful for detecting the clinical indicators of dementia, as described in Section 3 are recorded, with the view to further analysis to determine those most pertinent.

Activities are captured as a list of time-stamped events using a variety of technologies. Mouse/keyboard level detection utilises an imported .NET library ${ }^{1}$. At the operating system level, native C\# .NET libraries ${ }^{2}{ }^{3}$ are used to detect file system events (files changed, created and renamed) and changes to the clipboard. Microsoft UI Automation events ${ }^{4}$ are used to record events such as opening / closing / minimizing / maximizing windows, changes in focus, menus opened/closed and elements selected by the user. As the UI Automation framework hooks into all Windows operating system events which are abundant and fired constantly, often at a rapid rate, for example while the user is performing computer activities, decisions had to be made about which UIA events to record. For example, while the 'structure change' events appeared initially to be the richest source of information for monitoring user activities, these events eventually had to be ignored as listening for them resulted in performance issues along with gaps and/or delays in the logging of other events. At the application level, the Office Primary Interop Assemblies ${ }^{5}$ and the Internet Explorer automation object ${ }^{6}$ are used to detect events from Microsoft Word, Outlook and Internet Explorer, the three applications considered most relevant for monitoring activities of older adult users.

Further 'high level' events have been developed for the SAMS framework, derived from the low level data events described above. A mouse monitor has been created to read original mouse events, too abundant to be efficiently recorded and too low-level to be of use for later analysis, and aggregates these into mouse drags and mouse 'phases' (time periods between clicks or half second intervals), obtaining more useful information such as time, distance, and screen areas crossed. Similarly, key up and down events are paired and the code and duration are recorded. At the operating system level, mouse drag events are classified where possible into 'move', 'move into', 'resize' and 'scroll' events based on what is known about simultaneous low-level events(for example icon/window position or size changes, scroll and file

\footnotetext{
${ }^{1}$ ApplicationandGlobalMouseandKeyboardHooks.NetLibrary in C\#: http://globalmousekeyhook.codeplex. com/

2 FileSystemWatcher Class: https://msdn. microsoft.com/enus/library/system.io.

${ }^{3}$ Clipboard (.NET): https://msdn.microsoft.com/ enus/library/windows/desktop/ms648709(v= vs.85).aspx

${ }^{4}$ Microsoft UI Automation events: https://msdn. microsoft.com/enus/library/windows/ desktop/ee671221(v=vs.85).aspx

${ }^{5}$ Office Primary Interop Assemblies: https://msdn. microsoft.com/enus/library/15s06t57.aspx

${ }^{6}$ InternetExplorer object: https://msdn.microsoft. com/enus/library/aa752084(v=vs.85).aspx
} 
system events). In addition, UI Automation ${ }^{7}$ is used to maintain a map of the desktop including all window and icon positions. This map is used to derive higher level mouse move events, capturing moves into and out of icons or windows and to augment mouse event data with information such as the underlying icon/window name, position and display level.

The resulting log files are stored in the comma delimited (CSV) text format with each line/row representing an event. Within each (encrypted) line, the first column is the time and date of the event, the second column is the type of event (e.g. FOCUS_CHANGED, DESKTOP_DRAG_ENDED) and the final column is a set of semi-colon delimited name-value pairs (the exact set of values stored being dependent on the type of event). The use of CSV / text file format allows rapid storage of data (compared with storing in a database for example). The CSV format described is designed to be as compact as possible to minimize memory use, whilst remaining easy to parse later on, allowing interoperability between data cleaning and analysis tools.

The 'set tasks' experiment has been completed and manual checks have indicated that the monitoring tool has successfully recorded events as required. Initial results show some difference in distributions amongst the two groups for some of the event-types and these were found to be significant [23]. In the ongoing longitudinal study, six months of data has been recorded and successfully logged, encrypted, transmitted, decrypted, parsed and cleaned, allowing the analysis described in Section 3 B to commence.

\section{EVALUATION}

\section{A. 30-30 Set Tasks and Observations}

The set tasks experiment described in section III.B.2.A resulted in a set of log output files that could be matched against the set of tasks to evaluate the coverage of the monitoring tool. All 47 user sessions were manually checked and this confirmed that each of the 4 tasks (divided into 24 activities) was faithfully recorded. As an example, task 2 is displayed in Table I.

In addition, to further measure accuracy, a 'Participant Observation Notes' form was completed for each participant session by one of the facilitators (see Table II). This records when the participant required assistance or made mistakes. A set of 10 forms with the most detailed notes were selected from the 47 completed sessions.

The logs were again found to follow the tasks and observation notes closely. For example, in the first note shown in Table II for Task 3, Activity 3 (open the email with the subject 'Important Study' in a new window) the user is given instructions on how to open the email. This corresponds with a 33 second interval in the $\log$ files from the 'OUTLOOK_START_APP' event to the following 'OUTLOOK_READ_MAIL' event. This interval contains a pause (with no user activity) of 22 seconds followed by a double click: (represented by 2 consecutive

\footnotetext{
${ }^{7}$ Microsoft UI Automation: https://msdn. microsoft.com/enus/library/windows/ desktop/ee684009(v=vs.85).aspx
}

DESKTOP_MOUSE_DOWN and then MOUSE_UP events). This is followed within a second by an OUTLOOK_OPEN_MAIL event, signifying that the mail was opened in a new window. This event also contains an element name field with the value 'Important Study'.

Overall, the logs have been found to contain rich detail of user interaction across the desktop, Windows applications and browser activities, including email, with over $90 \%$ recall of known user activities.

\section{TABLE I. SAMPLE FROM 'PARTICIPANT INSTRUCTION SHEET'}

\begin{tabular}{|c|c|}
\hline & Task two - Email \\
\hline 1 & Start the email program ('Microsoft Outlook') \\
\hline 2 & $\begin{array}{l}\text { Find the email somebody has sent you, with the subject } \\
\text { 'Important-study' }\end{array}$ \\
\hline 3 & Open the email in a new window \\
\hline 4 & Close the email \\
\hline 5 & $\begin{array}{l}\text { Delete all the emails, with the word 'SPAM' in the title, from the } \\
\text { inbox. }\end{array}$ \\
\hline 6 & $\begin{array}{l}\text { Open the email, in the inbox, which has the subject 'Important: } \\
\text { Your participation' }\end{array}$ \\
\hline 7 & $\begin{array}{l}\text { Reply to this email, saying: "I am willing to take part in the } \\
\text { study", followed by your participant ID. } \\
\text { Send the email }\end{array}$ \\
\hline 8 & $\begin{array}{l}\text { Move the email located in the inbox, with the subject 'Study } \\
\text { Schedule', to the Outlook folder called 'SAMS' }\end{array}$ \\
\hline 9 & Close the email program ('Microsoft Outlook'). \\
\hline
\end{tabular}

TABLE II. SAMPLE FROM 'PARTICIPANT OBSERVATION NOTES'

\begin{tabular}{|c|c|c|}
\hline $\begin{array}{l}\text { Task } \\
\text { No. }\end{array}$ & Activity & Notes \\
\hline 2 & 3 & $\begin{array}{l}\text { Told how to open email in new window - "double } \\
\text { click" }\end{array}$ \\
\hline 2 & 5 & $\begin{array}{l}\text { Told to try and work it out "Go back to inbox" } \\
\text { Advised to look at top bar. }\end{array}$ \\
\hline 2 & 7 & "Try the top bar again" \\
\hline 2 & 7 & $\begin{array}{l}\text { "It's on the left" (Unsure where 'send' button was } \\
\text { located.) }\end{array}$ \\
\hline 2 & 8 & $\begin{array}{l}\text { Placed email in Junk - advised to try and correct. } \\
\text { Participant tried to drag junk folder to SAMS. } \\
\text { Advised how to correct - "drag email to SAMS" - } \\
\text { pointed to email on screen. }\end{array}$ \\
\hline
\end{tabular}

\section{B. Heat Maps}

The screen area's crossed data has been used to create mouse movement heat maps. A screen area is a $20 \times 20$ pixel sized square and any mouse moves with $\mathrm{X}$ and $\mathrm{Y}$ co-ordinates falling within the screen area increments the total count for that square. Similarly, the above events are also paired with mouse button down and mouse button up events using the SAMS collator tool, to produce a $2^{\text {nd }}$ set of heat-maps showing left and right click locations. As further evaluation of the monitoring output, these maps show a correspondence with expected mouse patterns for the applications that are used during the observed time periods. For example Fig. 2 and Fig 3 show heat-maps for mouse moves and mouse clicks respectively for a participant who regularly ran a solitaire application. The map shows data over a one week period. Similar results were found when observing other participants 
who regularly use other applications such as internet browsers and email.

Fig. 2. Mouse move heat-map for a solitaire user over a week

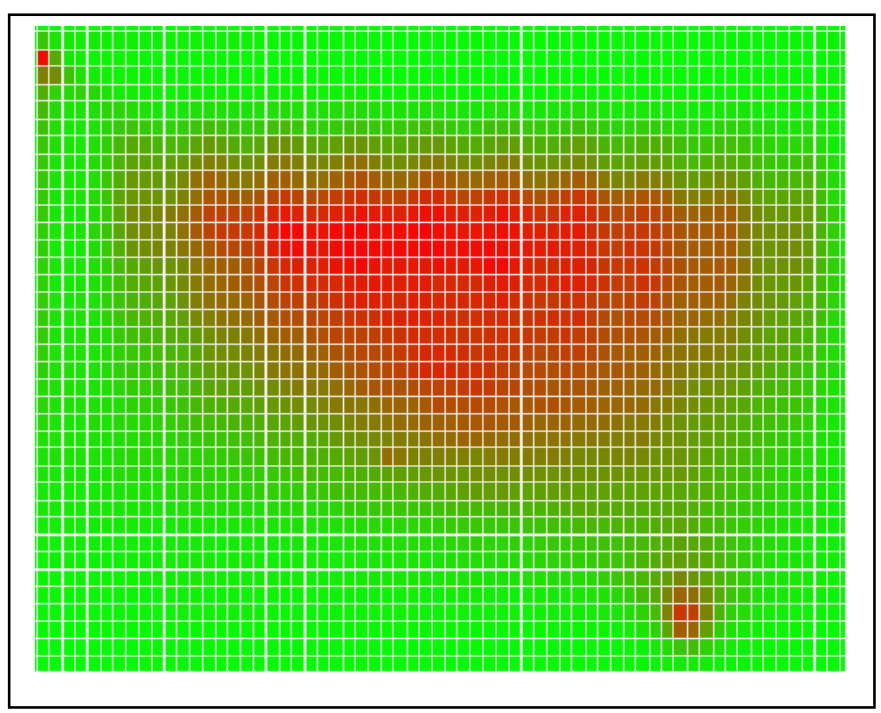

Fig. 3. Mouse left click heat-map for a solitaire user over a week.

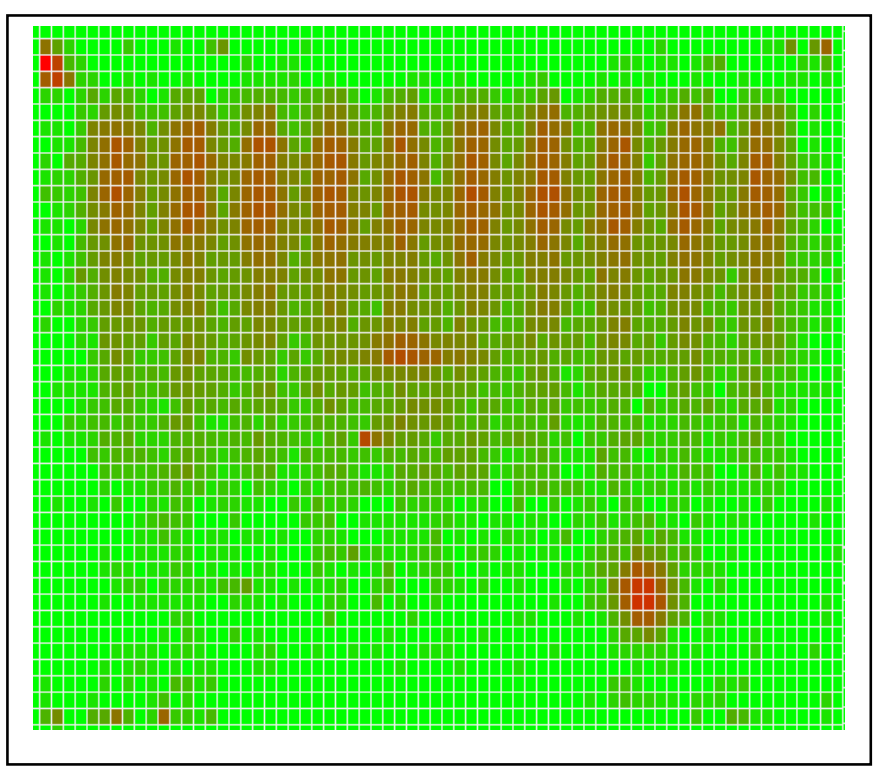

\section{Clinical Analysis: Early Findings}

The SAMS monitor logs have been used for sequence analysis and are found to be reliable for constructing frequent patterns of actions for each participant. The data is currently being analysed to establish a baseline of 'normal variation' i.e. change in user behaviour due to different Apps, time of day/week, etc. Once this baseline has been established within individuals and across the participant cohort, data/text mining will be applied to search for more subtle signs of mental health problems.

As required, the logger output is very detailed with a high coverage of user actions and related events. It therefore has the potential to be analysed with a variety of methods to establish baseline variation, e.g. event sequences, event frequencies/session/day/week, screen areas used from mouse move heat maps, event associations from transition matrices and cluster analysis.

Initial analysis has confirmed that differences in the results from monitored data correlate with clinical measures taken on healthy and MCI/Mild dementia participants [24]. For the set tasks experiment, the SAMS clinical team have used collated data derived from the raw output $\operatorname{logs}$ to discover significant differences in measures between the two cohorts, such as average durations of pauses (periods of user inactivity) and average key press durations. In addition, correlations between the paper-based cognitive test results and low level event data have been found.

\section{CONCLUSIONS}

A desktop monitoring application has been successfully developed that combines keyboard and mouse, desktop and application activities to discover differences in cognitive functioning amongst older computer users. This provides a set of requirements obtained from a variety of sources which include capture from domain experts, potential (elderly) endusers and data analysts, along with those obtained from the experiment design. The result is a system that has little impact on users' daily activities, ensures data security from the initial recording through to data analysis and records a variety of data deemed to be potentially useful for detecting activities corresponding to the clinical indicators of MCI. In addition, we have designed the tool to be generic (being potentially useful in other domains such as those listed in Section 2).

The monitoring tool has been evaluated using several approaches and has been found to record events accurately and showing patterns as expected. Work is almost complete on writing a data pre-processing tool that includes data parsing and cleaning and that uses JSON rules as input to allow for full flexibility. The pre-processing tool and rule sets can be used on any log files in the CSV format described in Section 4B above. As a result, the SAMS monitoring tool data are being cleaned, aggregated into the required higher level events, and early evidence and results indicate that the SAMS monitoring has the potential to be used to detect cognitive impairments.

\section{ACKNOWLEDGMENT}

The work described in this paper is funded by the Engineering and Physical Sciences Research Council (EPSRC) in the UK, within the Software Architecture for Mental Health Self-Management (SAMS) project, references EP/K015796/1, $\mathrm{EP} / \mathrm{K} 015761 / 1$ and EP/K015826/1. 


\section{REFERENCES}

[1] L. Lakey, K. Chandaria, C. Quince, M. Kane, and T. Saunders, "Dementia 2012: A national challenge," London: Alzheimer's Society, 2012.J. Clerk Maxwell, A Treatise on Electricity and Magnetism, 3rd ed., vol. 2. Oxford: Clarendon, 1892, pp.68-73.

[2] M. Kane and L. Cook, "Dementia 2013: The hidden voice of loneliness," London: Alzheimers Society, 2013.

[3] A. Burns and S. Iliffe, Dementia vol. 338: British Medical Journal, 2009.

[4] K. Zickuhr, "Generations 2010. Pew internet \& American life project," Pew Research Center, Washington, DC, 2010.

[5] K. Zickuhr and M. Madden, "Older adults and internet use," Pew Internet \& American Life Project, 2012.

[6] A. Seelye, S Hagler, N. Mattek, D. Howieson, K. Wild, H.H. Dodge, J. Kaye, "Computer mouse movement patterns: a potential marker of mild cognitive impairment", Alzheimer's \& Dementia, Assessment \& Disease Monitoring, 1-9, 2015

[7] H. Jimison, M. Pavel and J. McKanna, "Unobtrusive Computer Monitoring of Sensory Motor Function”, in Procs of the Intl Conference of the IEEE Engineering in Medicine and Biology Society, February 2005

[8] H. Jimison, N. Jessey, J. McKanna, T. Zitzelberger and J.Kaye, "Monitoring Computer Interactions to Detect Early Cognitive Impairment in Elders", In Procs of the $1^{\text {st }}$ Distributed Diagnosis and Home Healthcare conference, Arlington, USA, April 2006

[9] L. Giancardo, A. Sanchez-Ferro, I. Butterworth, C. S. Mendoza and J. M. Hooker, "Psychomotor Impairment Detection via Finger interactions with a computer keyboard during natural typing", Scientific Reports, Vol 5: 9678, 2015)

[10] P-M. Lee, W-H. Tsui and T-C. Hsiao, "The influence of emotion on keyboard typing: an experimental study using autidory stimuli", PLoS ONE, vol 10 (6): e0129056, 2015

[11] D. Carneiro, P. Novais, J. M. Pego, N. Sousa and J. Neves, "Using mouse dynamics to assess stress during online exams", E. Onieva et al (Eds.): HAIS, LNAI 9121, pp. 345-356, 2015

[12] S. Pachidi, M. Spruit, and I. Van De Weerd, "Understanding users' behavior with software operation data mining". Computers in Human Behavior, vol 30,pp. 583-594, 2014
[13] F. Meuller and A. Lockerd, "Cheese: tracking moues movement activity on websites, a tool for user modelling", In Proc.s of the Conference on Human Factors in Computing Systems (CHI), Seattle, USA, April 2001

[14] R. Atterer, M. Wnuk and A. Schmidt, "Knowing the user's every move - user activity tracking for website usability evaluation and implicit interaction", In Proc.s WWW conference, Edinburgh, UK, May 2006

[15] Q. Guo and E. Agichtein "Exploring mouse movements for inferring query intent", In Proc.s SIGIR, Singapore, July 2008

[16] J. Huang, R. W. White and S. Dumais, "No clicks, no problem: Using cursor movements to understand and improve search", In Proc.s of the Conference on Human Factors in Computing Systems (CHI), Vancouver, Canada, May 2011

[17] M. Pusara and C. E. Brodley, "User Re-authentication via mouse movements", In procs VizSec/DMSEC, Washington DC, USA, 2004

[18] L. C. F. Araujo, L. H. R. Sucupira Jr., M. G. Lizarraga, L. L. Ling and J. B. T. Yabu-Uti, "User Authentication through typing biometrics features, IEEE Transactions on Signal Processing, Vol 53, No. 2, February 2005

[19] Y. Deng and Y. Zhong "Keystroke dynamics user autentication based on gaussian mixture model and deep belief nets", Signal Processing, Hindawi Publishing Corporation, vol 2013

[20] A. Sutcliffe, P. Rayson, C. Bull and P. Sawyer, "Discovering affectladen requirements to achieve system acceptance", In procs of Requirements Engineering conference, Kariskrona, Sweden, 2014

[21] A. Sutcliffe et al, "Requirements elicitation with domain experts: Pitfalls and progress in healthcare", Unpublished (2016)

[22] A. Sutcliffe and P. Sawyer, "Requirements elicitation: towards the unknown unknowns", In procs Requirements Engineering conference, Rio de Janeiro, Brazil, 2013

[23] C. Bull, D. Asfiandy, A. Gledson, J. Mellor, S. Couth, G. Stringer, P. Rayson, A. Sutcliffe, J. Keane, X. Zeng, A. Burns, I. Leroi, C. Ballard and P. Sawyer, "Combining data mining and text mining for detection of early stage dementia: the SAMS framework", In Press, Language Resources and Evaluation Conference, Portorož, Slovenia, 2016.

[24] G. Stringer, L. Brown, D. Montaldi and I. Leroi, "Can you detect Alzheimer's from and email?", Postgraduate Summer Research Showcase, Manchester, 2016. 